\title{
Author Correction: ATF4 regulation of mitochondrial folate- mediated one-carbon metabolism is neuroprotective
}

\author{
Ivana Celardo ${ }^{1} \cdot$ Susann Lehmann ${ }^{1} \cdot$ Ana C. Costa ${ }^{1} \cdot$ Samantha H. Y. Loh ${ }^{1} \cdot$ L. Miguel Martins ${ }^{1}$
}

Published online: 13 December 2018

(c) ADMC Associazione Differenziamento e Morte Cellulare 2018

Correction to: Cell Death \& Differentiation (2017) 24: 638-648. https://doi.org/10.1038/cdd.2016.158, Published online 17 February 2017

Following publication of the article, Dr. Roberta Tufi of the Mitochondrial Biology Unit at the University of Cambridge was concerned to note that her own contribution to the study during her postdoc in Leicester at the MRC Toxicology Unit had not been acknowledged. Specifically, the data in Fig. 1 (panels a, b, and d) were produced though her work.

This correction acknowledges the significant time Dr. Tufi dedicated to the RNA preparations (the experimental protocols she performed and the raw data are visible publicly as they were deposited in ArrayExpress under accession no. E-MTAB-1406), and also to the growing, collection, and preparation of the samples for the metabolic analysis.

These authors contributed equally: Ivana Celardo, Susann Lehmann, Ana C. Costa

The original article can be found online at https://doi.org/10.1038/cdd. 2016.158 .

Samantha H. Y. Loh

shyl1@le.ac.uk

$\bowtie$ L. Miguel Martins

martins.lmiguel@gmail.com

1 MRC Toxicology Unit, Lancaster Road, Leicester LE1 9HN, UK 